\title{
Supernova Remnants in the Magellanic Clouds
}

\author{
M. D. Filipović ${ }^{1,2}$, L. Staveley-Smith ${ }^{2}$, W. Reid ${ }^{3}$, P. A. Jones ${ }^{2}$ \\ ${ }^{1}$ University of Western Sydney, Locked Bag 1797, Penrith South DC, \\ NSW 1797, Australia \\ ${ }^{2}$ Australia Telescope National Facility, CSIRO, P.O. Box 76, Epping, \\ NSW 1710, Australia \\ ${ }^{3}$ Department of Physics, Macquarie University, Sydney, NSW 2109, \\ Australia
}

\begin{abstract}
A study of the nature and the spatial distribution of over 250 discrete radio sources (H II regions and SNRs) in the Magellanic Clouds (MCs) is underway. A multi-frequency comparison of these sources involves the latest radio, IR, optical and X-ray surveys with angular resolution of $<1^{\prime}$. Special emphasis is given to the MC SNRs. Their morphology, birth rate and overall properties are investigated and compared with Galactic SNRs and SNRs from other nearby galaxies. Also, we are investigating the radio source distribution throughout both MCs.
\end{abstract}

\section{Introduction}

Statistical studies of SNRs in our Galaxy, despite their large number $(\sim 220)$, have problems due to incompleteness and uncertain distances. Objects in the MCs are approximately of similar distance and foreground absorption. However, they are still close enough to allow detailed spatially resolved investigations. The MCs contain all types of SNRs in various stages of evolution. Also, the MCs fortunately lie in a direction well out of the Galactic plane and the foreground densities of dust, gas and stars are small. SNRs in other galaxies such as M31 or M33 appear about 10 times smaller and 100 times fainter than MC SNRs. A comparison of radio and X-ray source flux densities shows very little correlation, but we note that the strongest SNRs at both frequencies are young SNRs from Population I. About 68 SNRs and SNR candidates in the LMC and 23 in the SMC have been identified in our recent studies. Of these, there are 29 new candidates in the LMC and 6 in the SMC which need to be confirmed.

\section{The ATCA Mosaic and Pointed Observations of SNRs in the MCs}

As a continuation of the Parkes radio-continuum investigations of sources intrinsic to the MCs and as an addition to the ATCA mosaic surveys, we observed most of these selected sources using the Compact Array of the ATNF at $\lambda=6$ and $3 \mathrm{~cm}$ with the $375 \mathrm{~m}$ configuration. These observations, together with the ATCA mosaic surveys of both Clouds at $20 / 13 \mathrm{~cm}$ and the MOST survey at $843 \mathrm{MHz}$, will give us wide frequency coverage of these objects with sufficient 
resolution to resolve them. The aim was to image a large number of SNRs and SNR candidates in "snap-shot" mode. The trade-off of image complexity with uv coverage indicates that this compromise of resolution is justified. Using this method we observed all SNRs and SNR candidates in both Clouds ( 90). Observations were undertaken during 12 observing days between 1997 and 2002 .

All SNR candidates from this study have a typical SNR characteristic. With spectral index $\alpha<-0.5 \pm 0.1$, we confirm them as SNRs. A general SNR-like morphology was detected in all other observed SNR objects. Typical "shell-like" structure can be seen in the majority of SNR candidates that were observed.

\section{Supernova Remnants in the SMC}

There are 23 known SNRs in the SMC. The ATCA Mosaic survey of the SMC detected 16 of these including 5 candidates. The selection of candidates should result in the most complete sample of small and intermediate sized SNRs. The Xray catalogs showed good alignment in matching the SNR radio sources. Strong $\mathrm{X}$-ray emission from sources is an efficient way for confirming SNRs. There is however a rapid reduction of apparent $\mathrm{X}$-ray luminosity with increasing size (Long 1983) which may result in many of the large diameter SNRs falling below the X-ray detection threshold. All 16 of our listed radio SNRs have also been detected in X-ray surveys. Three of our SNR candidates which have diameters of 50.7, 49.7 and $16.4 \mathrm{pc}$ have at least one listing in an X-ray catalog.

In our $\Sigma-D$ plot for the SMC SNRs it can be seen that there is a large degree of scatter in the sources. The error is approximately $10 \%$, however we find a relation of -1.5 and a mean slope of 0.4 . A more straightforward diagnostic of $\mathrm{SNR}$ evolution is the integral number-diameter $(N \propto D)$ relation. This relation holds true for Galactic SNRs which are heterogeneous and plagued with distance uncertainties. After calibrating distances, Mills (1983) obtains $N \propto D^{1.15}$. In the MCs, our initial estimates suggest a different phase of evolution for these SNRs compared to Galactic SNRs. The $N \propto D$ graph for the SMC SNRs and SNR candidates show that SMC SNRs of a diameter of $30 \mathrm{pc}$ are the most common, with a sharp fall-off in large diameter SNRs as expected.

\section{Future Work}

Our investigation of objects in the MCs are based on ATCA radio-continuum observations with the aim of studying a complete sample of MC SNRs. The luminosity-diameter distribution will be used to study the evolution of SNRs in a statistical sense. Comparisons with $\mathrm{H} \alpha$, [S II], [O III], XMM and Chandra surveys are planned. In addition, individual SNRs will be studied in more detail.

\section{References}

Long, K. S. 1983, in IAU Symp. 101, SNRs and their X-ray Emission, (Dordrecht: Reidel), p. 525

Mills, B. Y. 1983, in IAU Symp. 101, SNRs and their X-ray Emission, (Dordrecht: Reidel), p. 551 